\title{
Bortezomib-Based Therapy for Newly Diagnosed Mantle-Cell Lymphoma
}

\author{
Tadeusz Robak, M.D., Huiqiang Huang, M.D., Jie Jin, M.D., Jun Zhu, M.D., \\ Ting Liu, M.D., Olga Samoilova, M.D., Halyna Pylypenko, M.D., \\ Gregor Verhoef, M.D., Ph.D., Noppadol Siritanaratkul, M.D., \\ Evgenii Osmanov, M.D., Ph.D., Julia Alexeeva, M.D., Ph.D., Juliana Pereira, Ph.D., \\ Johannes Drach, M.D., Jiri Mayer, M.D., Xiaonan Hong, M.D., Rumiko Okamoto, M.D., \\ Lixia Pei, Ph.D., Brendan Rooney, Ph.D., Helgi van de Velde, M.D., Ph.D., \\ and Franco Cavalli, M.D., for the LYM-3002 Investigators*
}

ABSTRACT

The authors' affiliations are listed in the Appendix. Address reprint requests to Dr. Cavalli at the Oncology Institute of Southern Switzerland, Ospedale San Giovanni, $\mathrm{CH}-6500$ Bellinzona, Switzerland or at franco.cavalli@eoc.ch.

*A complete list of the LYM-3002 study investigators is provided in the Supplementary Appendix, available at NEJM.org.

N Engl J Med 2015;372:944-53. DOI: 10.1056/NEJMoal412096

Copyright $(2015$ Massachusetts Medical Society.

\section{BACKGROUND}

The proteasome inhibitor bortezomib was initially approved for the treatment of relapsed mantle-cell lymphoma. We investigated whether substituting bortezomib for vincristine in frontline therapy with R-CHOP (rituximab, cyclophosphamide, doxorubicin, vincristine, and prednisone) could improve outcomes in patients with newly diagnosed mantle-cell lymphoma.

\section{METHODS}

In this phase 3 trial, we randomly assigned 487 adults with newly diagnosed mantlecell lymphoma who were ineligible or not considered for stem-cell transplantation to receive six to eight 21-day cycles of R-CHOP intravenously on day 1 (with prednisone administered orally on days 1 to 5 ) or VR-CAP (R-CHOP regimen, but replacing vincristine with bortezomib at a dose of $1.3 \mathrm{mg}$ per square meter of body-surface area on days $1,4,8$, and 11). The primary end point was progression-free survival.

\section{RESULTS}

After a median follow-up of 40 months, median progression-free survival (according to independent radiologic review) was 14.4 months in the R-CHOP group versus 24.7 months in the VR-CAP group (hazard ratio favoring the VR-CAP group, 0.63; $\mathrm{P}<0.001$ ), a relative improvement of $59 \%$. On the basis of investigator assessment, the median durations of progression-free survival were 16.1 months and 30.7 months, respectively (hazard ratio, $0.51 ; \mathrm{P}<0.001$ ), a relative improvement of $96 \%$. Secondary end points were consistently improved in the VR-CAP group, including the complete response rate $(42 \%$ vs. $53 \%$ ), the median duration of complete response (18.0 months vs. 42.1 months), the median treatment-free interval (20.5 months vs. 40.6 months), and the 4 -year overall survival rate (54\% vs. $64 \%)$. Rates of neutropenia and thrombocytopenia were higher in the VR-CAP group.

CONCLUSIONS

VR-CAP was more effective than R-CHOP in patients with newly diagnosed mantlecell lymphoma but at the cost of increased hematologic toxicity. (Funded by Janssen Research and Development and Millennium Pharmaceuticals; LYM-3002 ClinicalTrials .gov number, NCT00722137.) 
ANTLE-CELL LYMPHOMA IS AN INCURable, aggressive hematologic cancer with a poor prognosis (median survival, 4 to 5 years). ${ }^{1,2}$ It comprises 5 to $6 \%$ of all nonHodgkin's lymphomas, including approximately 5000 cases per year in the United States. ${ }^{3}$ For previously untreated patients who are either ineligible or not considered for intensive chemotherapy and stem-cell transplantation, R-CHOP (rituximab, cyclophosphamide, doxorubicin, vincristine, and prednisone) is a standard of care ${ }^{4-6}$ and produces complete response rates of up to $48 \%$. However, progression-free survival is limited (median, 16.6 months). ${ }^{7}$

The proteasome inhibitor bortezomib was initially approved for the treatment of relapsed mantle-cell lymphoma in the United States ${ }^{8}$ and in 53 other countries on the basis of phase 2, single-agent data showing durable responses and favorable rates of progression-free survival and overall survival, with predictable toxic effects. ${ }^{9-11}$ In this phase 3 trial, LYM-3002, we investigated whether substituting bortezomib for vincristine (because of concern over potential overlapping neurotoxicity if the two drugs were administered in the same regimen) ${ }^{12}$ in frontline $\mathrm{R}-\mathrm{CHOP}$ could improve outcomes in patients with mantle-cell lymphoma. We assessed the efficacy and safety of R-CHOP versus combination therapy with bortezomib, rituximab, cyclophosphamide, doxorubicin, and prednisone (VR-CAP) in patients with newly diagnosed disease in whom stem-cell transplantation was not an option.

\section{METHODS}

\section{STUDY PATIENTS}

From May 2008 through December 2011, we enrolled patients at 128 sites in 28 countries across Europe, Asia, North America, and South America. Adults with newly diagnosed stage II, III, or IV mantle-cell lymphoma who were ineligible or not considered for stem-cell transplantation were eligible. (Complete eligibility criteria are provided in the Supplementary Appendix, available with the full text of this article at NEJM.org.) The diagnosis was confirmed on central pathological review.

The trial was conducted according to the provisions of the Declaration of Helsinki, Good Clinical Practice guidelines, and local regulatory requirements. The local ethics committee or institutional review board at each site approved the study protocol, which is available at NEJM.org. All patients provided written informed consent.

\section{STUDY OVERSIGHT}

The database lock for the primary analysis was January 10, 2014. The trial was designed by the academic authors in collaboration with employees of Janssen Research and Development, the sole legal sponsor of the study. Millennium Pharmaceuticals cofunded the study. Data were gathered by the investigators and Janssen employees, analyzed by the Janssen coauthors, and interpreted by the first and last authors, the academic coauthors, and the Janssen coauthors. The first and last authors and the Janssen coauthors vouch for the integrity, accuracy, and completeness of the data analyses and for the fidelity of the study to the protocol. The initial draft was written by the first and last authors, the Janssen coauthors, and a medical writer employed by FireKite and paid by Janssen and Millennium Pharmaceuticals. All authors were involved in manuscript development and approved the final draft. The decision to submit the manuscript for publication was made by the first and last authors and agreed by the coauthors.

\section{STUDY TREATMENTS}

Patients were stratified according to their score on the International Prognostic Index (IPI), with risks categorized as low (a score of 0 or 1 ), lowintermediate (a score of 2), high-intermediate (a score of 3), or high (a score of 4 or 5) (Table S1 in the Supplementary Appendix), and disease stage at diagnosis (stage II, III, or IV according to the staging system for non-Hodgkin's lymphoma of the American Joint Committee on Cancer). Patients were randomly assigned in a 1:1 ratio to receive six 21-day cycles of $\mathrm{R}-\mathrm{CHOP}$ or VR-CAP. Patients could receive up to eight cycles if a response was first documented at cycle 6. R-CHOP comprised rituximab (at a dose of $375 \mathrm{mg}$ per square meter of body-surface area), cyclophosphamide (750 mg per square meter), doxorubicin (50 mg per square meter), and vincristine (1.4 mg per square meter, with a maximum total dose of $2 \mathrm{mg}$, all administered intravenously on day 1 , plus oral prednisone (100 mg per square meter) administered on days 1 to 5 . VR-CAP comprised 
intravenous bortezomib (1.3 mg per square meter) on days $1,4,8$, and 11 of each cycle (administered first on day 1), followed by rituximab (administered second on day 1) and cyclophosphamide, doxorubicin, and prednisone, all as described above.

All study drugs were provided from a central supply and paid for by the study budget. A permuted-block central randomization plan with a computer-generated randomization schedule (sponsor-generated) was used. Concomitant treatments and prophylactic medications are summarized in the Methods section in the Supplementary Appendix. Dose adjustments for toxic effects were permitted with the use of established dose-modification guidelines per the prescribing information for each drug.

\section{STUDY END POINTS AND ASSESSMENTS}

The primary end point was progression-free survival. The primary analysis was based on a blinded assessment of disease progression that was performed by an independent radiology review committee. Progression-free survival according to investigator assessment was used as supportive evidence. Prespecified secondary end points included the overall response rate (complete response or unconfirmed complete response and partial response), complete response rate (radiologic complete response or radiologic unconfirmed complete response, which both had to be verified by evidence of bone marrow clearance and normalization of the lactate dehydrogenase level), time to and duration of response, time to progression, time to next antilymphoma therapy, overall survival, and safety. All secondary end points are listed in the Methods section in the Supplementary Appendix.

Computed tomographic (CT) scans were performed every two cycles during treatment and every 6 to 8 weeks during follow-up until disease progression, study discontinuation, initiation of alternative therapy, or death. All CT results were assessed in a blinded fashion by the independent review committee and by investigators, according to the modified criteria of the International Workshop to Standardize Response Criteria for Non-Hodgkin's Lymphoma (Table S2 in the Supplementary Appendix). ${ }^{13}$ Adverse events were graded according to the National Cancer Institute Common Terminology Criteria for Adverse
Events, version 3.0. ${ }^{14}$ A central immunohistochemical analysis was performed to assess the expression of the prognostic marker $\mathrm{Ki}-67^{15-18}$ with the use of paraffin-embedded, formalinfixed, or frozen tumor samples (see the Supplementary Appendix for details). Ki-67-positive status was defined as an expression of more than $10 \%$ on an ordinal scale (the accepted cutoff for prognostic significance). ${ }^{15}$

\section{STATISTICAL ANALYSIS}

An independent data and safety monitoring committee oversaw the conduct of the study. It was estimated that 295 events of disease progression or death would provide a power of $80 \%$ (at a twosided alpha level of 0.05 ) to detect a $40 \%$ improvement in the median progression-free survival (from 18 to 25 months) with VR-CAP, as compared with R-CHOP. Assuming a data-accrual period of 24 months and 18 months of followup, we determined that 486 patients (243 per study group) were required. Three preplanned interim analyses were conducted.

All primary and secondary efficacy analyses were performed in the intention-to-treat population, except for response end points (which were analyzed in the response-evaluable population) and treatment-free interval (which was analyzed in the safety population). (Details regarding the analysis populations are provided in the Methods section in the Supplementary Appendix.)

We used Kaplan-Meier methods to estimate time-to-event distributions, with stratified logrank tests and Cox models used for betweengroup comparisons of time-to-event end points. We conducted prespecified subgroup analyses of progression-free survival according to IPI risk score, sex, race, region, age, disease stage at diagnosis, performance status (according to Eastern Cooperative Oncology Group criteria), lactate dehydrogenase level, and white-cell count.

We also performed post hoc analyses of progression-free survival according to mantle-cell lymphoma-specific IPI (MIPI) risk category, ${ }^{19,20}$ Ki-67 expression status ( $\leq 10 \%$ vs. $>10 \%$ ), and MIPI with biologic component (MIPIb) ${ }^{19,20}$ risk category in patients with baseline Ki-67 assessment. We used a stratified Cochran-MantelHaenszel chi-square test with IPI and disease stage as stratification factors to assess betweengroup differences in response rates. 


\section{RESULTS}

\section{PATIENTS}

A total of 487 patients underwent randomization to receive either R-CHOP (244 patients) or VR-CAP (243 patients) (Fig. S1 in the Supplementary Appendix). The diagnosis of mantle-cell lymphoma was confirmed by central pathological review in
471 patients ( $97 \%$ concordance). Demographic and disease characteristics were generally well balanced in the two groups (Table 1, and Table S3 in the Supplementary Appendix). Betweengroup distributions according to baseline Ki-67 expression and MIPIb risk category were similar (Table S4 in the Supplementary Appendix).

Overall, 406 patients (83\%) in the two study

\begin{tabular}{|c|c|c|c|}
\hline Variable & $\begin{array}{l}\text { R-CHOP } \\
(\mathrm{N}=244)\end{array}$ & $\begin{array}{l}\text { VR-CAP } \\
(N=243)\end{array}$ & $\begin{array}{l}\text { All Patients } \\
(\mathrm{N}=487)\end{array}$ \\
\hline \multicolumn{4}{|l|}{ Age } \\
\hline Median (range) $-y r$ & $66(34-82)$ & $65(26-88)$ & $66(26-88)$ \\
\hline$\geq 60 \mathrm{yr}-$ no. $(\%)$ & $177(73)$ & $178(73)$ & $355(73)$ \\
\hline Male sex - no. (\%) & $182(75)$ & $178(73)$ & $360(74)$ \\
\hline \multicolumn{4}{|l|}{ Race - no. $(\%) \dagger$} \\
\hline White & $172(70)$ & $151(62)$ & $323(66)$ \\
\hline Asian & $68(28)$ & $88(36)$ & $156(32)$ \\
\hline Other & $4(2)$ & $4(2)$ & $8(2)$ \\
\hline \multicolumn{4}{|l|}{ MIPI risk category — no. (\%) } \\
\hline Low & $70(29)$ & $76(31)$ & $146(30)$ \\
\hline Intermediate & $93(38)$ & $96(40)$ & $189(39)$ \\
\hline High & $80(33)$ & $71(29)$ & $151(31)$ \\
\hline Missing data & $1(<1)$ & 0 & $1(<1)$ \\
\hline \multicolumn{4}{|l|}{ Disease stage at diagnosis — no. (\%) } \\
\hline II & $16(7)$ & $12(5)$ & $28(6)$ \\
\hline III & $42(17)$ & $49(20)$ & $91(19)$ \\
\hline IV & $186(76)$ & $182(75)$ & $368(76)$ \\
\hline Elevated lactate dehydrogenase - no. (\%) & $86(35)$ & $88(36)$ & $174(36)$ \\
\hline Bone marrow involvement — no. (\%) & $171(70)$ & $165(68)$ & $336(69)$ \\
\hline Extranodal involvement - no. (\%) & $137(56)$ & $139(57)$ & $276(57)$ \\
\hline \multicolumn{4}{|l|}{ Histologic subtype — no./total no. (\%) } \\
\hline Blastoid & $28 / 239(12)$ & $25 / 236(11)$ & $53 / 475(11)$ \\
\hline Nodular & $97 / 239(41)$ & $109 / 236(46)$ & $206 / 475(43)$ \\
\hline \multicolumn{4}{|c|}{ Reason for ineligibility for stem-cell transplantation - no. (\%) $\pitchfork \delta$} \\
\hline Age $\geq 60$ yr or medically ineligible & $202(83)$ & $205(84)$ & $407(84)$ \\
\hline Age $<60 \mathrm{yr}$ and not considered for transplantation & $42(17)$ & $38(16)$ & $80(16)$ \\
\hline
\end{tabular}

* There were no significant differences between the groups in the listed categories, except for race $(P=0.03)$. Percentages may not total 100 because of rounding. MIPI denotes mantle-cell lymphoma-specific International Prognostic Index; R-CHOP rituximab, cyclophosphamide, doxorubicin, vincristine, and prednisone; and VR-CAP bortezomib, rituximab, cyclophosphamide, doxorubicin, and prednisone.

Race was determined by investigators.

The assessment was performed by the sponsor's medical monitor.

$\int$ Most patients were enrolled on the basis of ineligibility for transplantation owing to medical reasons (age, $\geq 60$ years; or the presence of coexisting medical conditions, in accordance with current protocols). ${ }^{21}$ Patients were not considered for transplantation because of socioeconomic reasons (financial affordability), their decision not to undergo the procedure, or lack of transplant availability. 


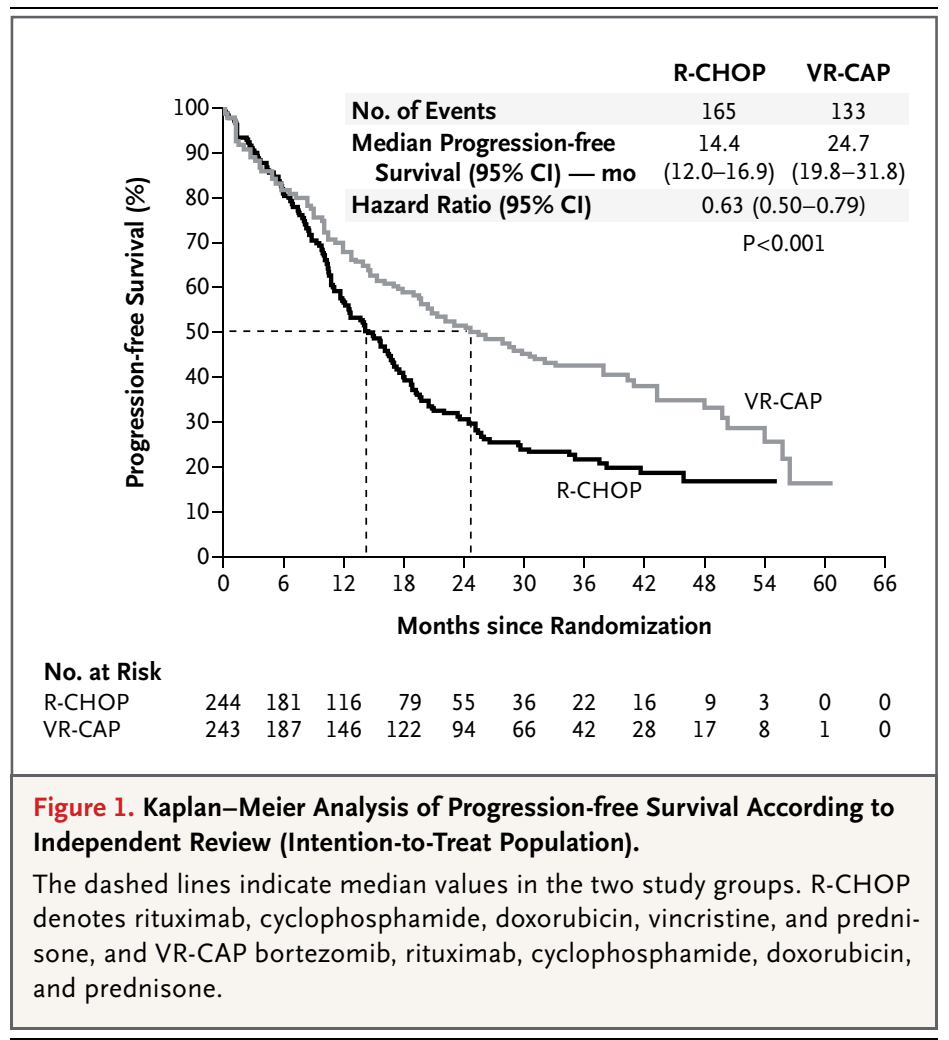

groups (203 per group) received six or more cycles of a study drug (median, 6 [range, 1 to 8] in the two groups). Treatment exposure was similar in the two groups, and most patients received the planned doses of each drug (Table 55 in the Supplementary Appendix). The mean relative dose intensity for drugs common to both regimens was $93 \%$ or higher. The mean relative dose intensity was $80 \%$ for vincristine in the R-CHOP group (owing to the dose capping at $2 \mathrm{mg}$ ) and $82 \%$ for bortezomib in the VR-CAP group.

\section{PRIMARY END POINT}

After a median follow-up of 40 months (40.3 months in the R-CHOP group and 39.4 months in the VR-CAP group), 298 patients (61\%) had disease progression or died, according to the independent review committee. The median progression-free survival was 14.4 months in the R-CHOP group and 24.7 months in the VR-CAP group (hazard ratio favoring the VR-CAP group, 0.63; $\mathrm{P}<0.001$ ) (Fig. 1). This result represented a relative improvement of $59 \%$ in the VR-CAP group, which exceeded the hypothesized $40 \%$ improvement. According to the investigator assessment, 307 patients $(63 \%)$ had disease progression or died, and the median progression-free survival was 16.1 months in the R-CHOP group and 30.7 months in the VR-CAP group (hazard ratio, 0.51; $\mathrm{P}<0.001$ ), representing a $96 \%$ improvement (Fig. S2 in the Supplementary Appendix).

Preplanned sensitivity analyses showed that the difference between the assessments of the independent review committee and the investigators was predominantly driven by the conservative assessments of the independent review committee of progression with respect to transient fluid collections or transient lesions in patients who had a subsequent response or stable disease (Table S6 in the Supplementary Appendix). There was a consistent improvement in the VR-CAP group with respect to progression-free survival on the basis of baseline characteristics across all prespecified subgroups (Fig. S3 in the Supplementary Appendix). Furthermore, VR-CAP had a consistent, significant effect on progression-free survival in both Ki-67-positive patients and in those with either no or low Ki-67 expression and across all MIPIb risk categories (Table S7 in the Supplementary Appendix).

\section{RESPONSE RATE}

Most patients had a tumor response according to the assessment of the independent review committee (Table 2). Rates of complete response were significantly lower in the R-CHOP group than in the VR-CAP group ( $42 \%$ vs. $53 \%$ ). According to the independent assessment, in the R-CHOP group, as compared with the VR-CAP group, the median time to response was 1.6 months versus 1.4 months, the median duration of overall response was 15.1 months versus 36.5 months, and the median duration of complete response was 18.0 months versus 42.1 months. Improvements in response rates and durability of response in the VR-CAP group, as compared with the R-CHOP group, were also observed for investigatorassessed responses (Table S8 in the Supplementary Appendix).

\section{OTHER SECONDARY END POINTS}

For R-CHOP versus VR-CAP, the median time to progression by independent assessment was 16.1 months versus 30.5 months (hazard ratio, 0.58), the median time to the next antilymphoma therapy was 24.8 months versus 44.5 months (hazard ratio, 0.50), and the median treatment-free interval was 20.5 months versus 40.6 months (hazard 


\begin{tabular}{|c|c|c|c|}
\hline End Point & R-CHOP & VR-CAP & $\begin{array}{c}\text { Hazard Ratio or Risk Ratio } \\
(95 \% \mathrm{Cl}) \dagger\end{array}$ \\
\hline \multicolumn{4}{|l|}{ Best response rate on independent review } \\
\hline No. of patients evaluated & 228 & 229 & \\
\hline Overall response - no. (\%) & $204(89)$ & $211(92)$ & $1.03(0.97-1.09)$ \\
\hline Complete response - no. (\%)』 & $95(42)$ & $122(53)$ & $1.29(1.07-1.57) 9$ \\
\hline \multicolumn{4}{|l|}{ Time to response on independent review } \\
\hline No. of patients evaluated & 228 & 229 & \\
\hline Median - mo & 1.6 & 1.4 & $1.54(1.26-1.89) \|$ \\
\hline \multicolumn{4}{|l|}{ Duration of response on independent review } \\
\hline \multicolumn{4}{|l|}{ Overall response } \\
\hline No. of patients evaluated & 204 & 211 & \\
\hline Median - mo $(95 \% \mathrm{Cl})$ & $15.1(12.5-17.0)$ & $36.5(26.7-46.7)$ & NA \\
\hline \multicolumn{4}{|l|}{ Complete response } \\
\hline No. of patients evaluated & 95 & 122 & \\
\hline Median - mo $(95 \% \mathrm{Cl})$ & $18.0(14.0-23.4)$ & $42.1(30.7-49.1)$ & NA \\
\hline \multicolumn{4}{|l|}{ Time to progression } \\
\hline No. of patients evaluated & 244 & 243 & \\
\hline Median on independent review - mo & 16.1 & 30.5 & $0.58(0.45-0.74) \|$ \\
\hline Median on investigator review - mo & 16.8 & 35.0 & $0.47(0.36-0.60) \|$ \\
\hline \multicolumn{4}{|l|}{ Time to next antilymphoma treatment } \\
\hline No. of patients evaluated & 244 & 243 & \\
\hline Median - mo & 24.8 & 44.5 & $0.50(0.38-0.65) \|$ \\
\hline \multicolumn{4}{|l|}{ Treatment-free interval } \\
\hline No. of patients evaluated & 242 & 240 & \\
\hline Median - mo & 20.5 & 40.6 & $0.50(0.38-0.65) \|$ \\
\hline \multicolumn{4}{|l|}{ Overall survival } \\
\hline No. of patients evaluated & 244 & 243 & \\
\hline Median - mo & 56.3 & NR & $0.80(0.59-1.10)$ \\
\hline Survival rate at $4 \mathrm{yr}-\%(95 \% \mathrm{Cl})$ & $54(45-62)$ & $64(56-71)$ & NA \\
\hline
\end{tabular}

$* \mathrm{Cl}$ denotes confidence interval, NA not applicable, and NR not reached.

$\dagger$ Hazard ratios were calculated for time-to-event outcomes and risk ratios for response rates.

$\mp$ Included in the category of overall response were patients who had a complete response or an unconfirmed complete response plus a partial response, regardless of verification by means of bone marrow assessment or measurement of the lactate dehydrogenase level.

$\int$ Included in the category of complete response were patients who had a radiologic complete response or radiologic unconfirmed complete response, which both had to be verified by evidence of bone marrow clearance and normalization of the lactate dehydrogenase level.

If $P=0.007$.

$\| \mathrm{P}<0.001$.

ratio, 0.50) (Table 2). At the final analysis, $132 \mathrm{pa-}$ tients (54\%) in the R-CHOP group and 82 patients (34\%) in the VR-CAP group had received subsequent antilymphoma therapy (Table S9 in the Supplementary Appendix); of these patients, 67 (51\%) and 32 (39\%), respectively, had received two or more lines of therapy. The type of subsequent therapy was generally similar in the two groups, with 25 patients (19\%) in the R-CHOP group and $3(4 \%)$ in the VR-CAP group receiving subsequent bortezomib.

After the deaths of 158 patients (32\%), overall 


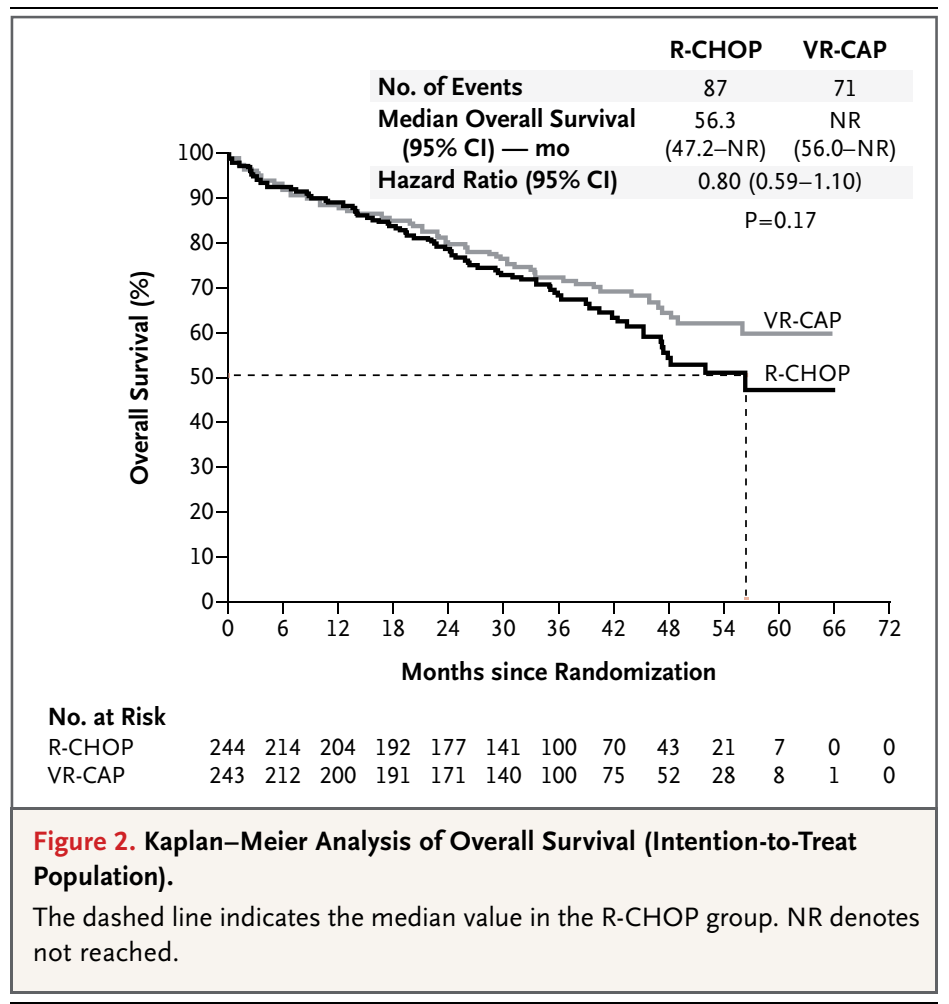

survival data were not mature at the time of this report. The median overall survival was 56.3 months in the R-CHOP group and had not been reached in the VR-CAP group (hazard ratio, 0.80; $\mathrm{P}=0.17$ ) (Fig. 2). There was a between-group difference in 4-year survival of 10 percentage points (54\% in the R-CHOP group vs. $64 \%$ in the VRCAP group).

\section{SAFETY}

Rates of adverse events of any grade and discontinuations due to adverse events were similar in the R-CHOP group and the VR-CAP group, including rates of drug-related adverse events $(93 \%$ vs. $96 \%$ ) and discontinuations (6\% vs. $8 \%$ ) (Table S10 in the Supplementary Appendix). Patients in the R-CHOP group, as compared with the VR-CAP group, had lower rates of grade 3 or higher adverse events (all events, $85 \%$ vs. $93 \%$; drug-related events, $80 \%$ vs. $91 \%$ ) and serious adverse events (all events, 30\% vs. 38\%; drug-related events, $21 \%$ vs. $32 \%)$.

Hematologic toxic effects were the most common adverse events (Table 3). The lower rates of thrombocytopenia that were observed in the $\mathrm{R}-\mathrm{CHOP}$ group, as compared with the VR-CAP group, were reflected in a lower rate of platelet transfusions ( $3 \%$ vs. $23 \%$ ). In the VR-CAP group, 48 of 54 patients $(89 \%)$ received platelet transfusions during days 10 to 14 of the treatment cycles. Ten of 13 patients $(77 \%)$ in the VR-CAP group who had the worst platelet counts $(<10,000$ per cubic millimeter) received platelet transfusions (Table S11 in the Supplementary Appendix). Thrombocytopenia led to a delay in next-cycle administration in $2 \%$ of patients in the R-CHOP group and 5\% of those in the VR-CAP group. Rates of bleeding events were similar in the two groups (any grade, 12 patients [5\%] vs. 15 patients [6\%]; grade $\geq 3,3$ patients [1.3\%] vs. 4 patients [1.6\%]). Rates of neutropenia and infections or infestations were lower in the R-CHOP group than in the VR-CAP group (Table 3), consistent with reduced use of colony-stimulating factors $(61 \%$ vs. $78 \%$ ) and systemic antibacterial agents (65\% vs. $81 \%$ ) (Table S11 in the Supplementary Appendix). Peripheral neuropathy rates were 29\% in the R-CHOP group and 30\% in the VR-CAP group (grade $\geq 3,4 \%$ vs. $8 \%$ ). Peripheral neuropathy was reversible in the majority of patients, with a complete resolution rate of $75 \%$ in the $\mathrm{R}-\mathrm{CHOP}$ group and $81 \%$ in the VR-CAP group, in a median of 5.5 months and 3.0 months, respectively (Table S11 in the Supplementary Appendix).

Rates of individual serious adverse events were less than $5 \%$ in each group, except for lower rates in the R-CHOP group than in the VR-CAP group for febrile neutropenia ( $8 \%$ vs. $11 \%$ ), neutropenia ( $5 \%$ each), and pneumonia ( $3 \%$ vs. $8 \%$ ). Fatal events during treatment occurred in 14 patients $(6 \%)$ in the R-CHOP group and 11 patients (5\%) in the VR-CAP group; of these deaths, 7 (3\%) and $5(2 \%)$, respectively, were due to drug-related adverse events: infection (in 3 patients), cardiac failure (in 2 patients), hypotension, diarrhea, and renal failure (in 1 patient), and tumor lysis syndrome (in 1 patient) in the R-CHOP group and infection (in 3 patients), cardiac failure (in 1 patient), and pulmonary embolism (in 1 patient) in the VR-CAP group (Table S10 in the Supplementary Appendix).

\section{DISCUSSION}

LYM-3002 was a randomized, phase 3 study incorporating bortezomib into frontline treatment in patients with newly diagnosed mantle-cell lymphoma. The study enrolled 487 patients with 
baseline characteristics that were generally consistent with those reported in other large studies $^{21-24}$ and with those in the broader population of patients with mantle-cell lymphoma. ${ }^{20}$

The study met its primary end point by showing a 59\% improvement in median progressionfree survival with VR-CAP versus R-CHOP (hazard ratio, 0.63; $\mathrm{P}<0.001)$. Consistently, investigator assessment of progression-free survival also favored VR-CAP, with 96\% improvement (hazard ratio, $0.51 ; \mathrm{P}<0.001)$. Progression-free survival for patients receiving R-CHOP was at the lower end of the range that has been reported previously, ${ }^{7,21-24}$ reflecting stringent and frequent CT tumor assessment (every 6 to 8 weeks).

Subgroup analyses of progression-free survival indicated the superiority of VR-CAP over R-CHOP regardless of baseline characteristics. Progression-free survival also appeared to be more favorable regardless of Ki-67 expression status (an established prognostic marker in mantle-cell lymphoma). ${ }^{15-18}$ The shorter median progression-free survival observed in Ki-67-positive patients, as compared with patients with no or low $\mathrm{Ki}-67$ expression, in the two study groups is consistent with the established negative prognostic cutoff value of Ki-67 expression of more than $10 \% .{ }^{15}$ When progression-free survival was analyzed according to MIPIb risk category (a validated disease-specific prognostic index, which became available for prognostic stratification after commencement of LYM-3002), ${ }^{19,20}$ the effect of VR-CAP was most apparent in the low- and intermediate-risk categories.

Significant improvements in secondary efficacy end points were shown for VR-CAP versus R-CHOP after a relatively short treatment duration (median, 4.0 months for VR-CAP), including higher rates of complete response, which were more durable in the VR-CAP group than in the R-CHOP group (median duration, 3.5 years vs. 1.5 years). There was a doubling of time to disease progression and time to the next antilymphoma treatment among patients receiving VR-CAP, as compared with those receiving R-CHOP. Patients in the VR-CAP group also benefited from an additional median period of 1.7 years without treatment. Although no significant difference in median overall survival was observed at the time of this report, a trend toward improved survival in the VR-CAP group (hazard ratio, $0.80 ; \mathrm{P}=0.17$ ) plus a difference in 4-year survival of 10 percent-

\begin{tabular}{|c|c|c|c|c|}
\hline \multirow[t]{3}{*}{ Adverse Event } & \multicolumn{2}{|c|}{ R-CHOP (N=242) } & \multicolumn{2}{|c|}{ VR-CAP $(N=240)$} \\
\hline & Any Grade & Grade $\geq 3$ & Any Grade & Grade $\geq 3$ \\
\hline & \multicolumn{4}{|c|}{ no. of patients (\%) } \\
\hline Any event & $238(98)$ & $206(85)$ & 238 (99) & $223(93)$ \\
\hline \multicolumn{5}{|l|}{ Hematologic event } \\
\hline Neutropenia & $178(74)$ & $162(67)$ & $211(88)$ & $203(85)$ \\
\hline Thrombocytopenia & $46(19)$ & $14(6)$ & $173(72)$ & $136(57)$ \\
\hline Anemia & $90(37)$ & $33(14)$ & $122(51)$ & $37(15)$ \\
\hline Leukopenia & $93(38)$ & $71(29)$ & $120(50)$ & $105(44)$ \\
\hline Lymphocytopenia & $32(13)$ & $21(9)$ & $74(31)$ & $67(28)$ \\
\hline Febrile neutropenia & $34(14)$ & $33(14)$ & $41(17)$ & $36(15)$ \\
\hline \multicolumn{5}{|l|}{ Gastrointestinal event } \\
\hline Diarrhea & $22(9)$ & $5(2)$ & $73(30)$ & $12(5)$ \\
\hline Constipation & $38(16)$ & $2(1)$ & $60(25)$ & $1(<1)$ \\
\hline Nausea & $33(14)$ & 0 & $59(25)$ & $1(<1)$ \\
\hline \multicolumn{5}{|l|}{ Infection or infestation } \\
\hline Any & $112(46)$ & $33(14)$ & $143(60)$ & $51(21)$ \\
\hline Pneumonia & $15(6)$ & $11(5)$ & $28(12)$ & $17(7)$ \\
\hline \multicolumn{5}{|l|}{ Nervous system disorder } \\
\hline $\begin{array}{l}\text { Peripheral neuropathy } \\
\text { not elsewhere } \\
\text { classified } †\end{array}$ & $69(29)$ & $10(4)$ & $73(30)$ & $18(8)$ \\
\hline $\begin{array}{c}\text { Peripheral sensory } \\
\text { neuropathy }\end{array}$ & $48(20)$ & $6(2)$ & $54(22)$ & $12(5)$ \\
\hline \multicolumn{5}{|l|}{ Other condition } \\
\hline Pyrexia & $37(15)$ & $5(2)$ & $70(29)$ & $8(3)$ \\
\hline Fatigue & $47(19)$ & $6(2)$ & $56(23)$ & $15(6)$ \\
\hline Cough & $20(8)$ & 0 & $49(20)$ & $3(1)$ \\
\hline Decreased appetite & $23(10)$ & $2(1)$ & $46(19)$ & $2(1)$ \\
\hline Asthenia & $26(11)$ & $2(1)$ & $38(16)$ & $7(3)$ \\
\hline Peripheral edema & $25(10)$ & $1(<1)$ & 37 (15) & $1(<1)$ \\
\hline
\end{tabular}

* The safety population included all the patients who underwent randomization and who received at least one dose of a study drug. Listed are adverse events of any grade that were reported in at least $15 \%$ of the patients and events of grade 3 or higher that were reported in at least $5 \%$ of the patients in either study group during the treatment period. Incidence is based on the number of patients with at least one adverse event. In addition to the listed events, alopecia, insomnia, neuralgia, stomatitis, and vomiting of any grade were reported in at least $10 \%$ but less than $15 \%$ of the patients in the VR-CAP group. $\uparrow$ This category includes the following preferred terms: peripheral sensory neuropathy, neuropathy peripheral, peripheral motor neuropathy, and peripheral sensorimotor neuropathy.

age points were noted, even though only $32 \%$ of patients had died. Longer follow-up will be required to show whether the Kaplan-Meier curves will continue to separate. Subsequent therapies 
were generally similar for patients with disease progression in the two study groups.

A limitation of our study is that rituximab maintenance therapy was not used; current recommendations ${ }^{4,6}$ were not established when the study began. The use of VR-CAP with rituximab maintenance therapy could further prolong progression-free survival and potentially extend overall survival.

The improved efficacy of VR-CAP over R-CHOP was accompanied by additional toxic effects. However, there was no significant effect on the number of completed cycles, median dose intensity for drugs common to both regimens, or rates of discontinuations or deaths related to adverse events. The VR-CAP safety profile was expected on the basis of previous experience with bortezomib plus R-CHOP in non-Hodgkin's lymphomas $^{25-27}$ and with single-agent bortezomib in relapsed or refractory mantle-cell lymphoma. ${ }^{9-11,28}$ Previous experience from a noncomparative phase 2 study of weekly or twice-weekly bortezomib plus R-CHOP suggested that weekly administration of bortezomib may be associated with reduced rates of some grade 3 or 4 hematologic and nonhematologic toxic effects but also with a reduced rate of complete response. ${ }^{25}$

Rates of peripheral neuropathy, a known toxicity associated with bortezomib and vincristine, ${ }^{8-11,22,24,28,29}$ were similar in the two groups, and events appeared to resolve more quickly in the VR-CAP group. Peripheral neuropathy rates also compared favorably with those reported in trials involving patients with multiple myeloma (ranging from $37 \%$ to $44 \%$ ). ${ }^{30}$ Bortezomib was administered intravenously in our study, but subcutaneous administration may offer improved safety, including a reduced risk of peripheral neuropathy, ${ }^{31}$ with added patient convenience. ${ }^{32}$

Rates of thrombocytopenia were increased in the VR-CAP group, but there was no betweengroup difference in rates of clinically significant bleeding events, similarly low rates of cycle delays due to thrombocytopenia, and no trend toward cumulative toxicity on thrombopoiesis, reflecting the previously reported transient, cyclical nature of platelet-count reduction with bortezomib. ${ }^{33-36}$ Notably, supportive therapies, which were allowed according to the protocol, were a key aspect of patient treatment in this study. The higher rate of platelet transfusion in the VR-CAP group may have been associated with the aim of maximizing bortezomib dose intensity; data indicate that the primary use of platelet transfusion was prophylactic (as allowed according to the protocol at the investigators' discretion) to avoid withholding the bortezomib dose on day 11 because of a platelet count of less than 25,000 per cubic millimeter, rather than therapeutic, because of a platelet count of less than 10,000 per cubic millimeter.

In conclusion, a significant prolongation of progression-free survival and improvements in secondary efficacy end points were observed with VR-CAP as compared with R-CHOP. This improvement was accompanied by additional, predominantly hematologic toxicity.

Supported by Janssen Research and Development and Millennium Pharmaceuticals, a wholly owned subsidiary of Takeda Pharmaceutical.

Disclosure forms provided by the authors are available with the full text of this article at NEJM.org.

We thank the patients who participated in this study and their families; the staff members at all the clinical sites; and Emma Landers, a medical writer employed by FireKite, for providing writing support during the development of the manuscript.

\section{APPENDIX}

The authors' affiliations are as follows: the Department of Hematology, Medical University of Lodz, Copernicus Memorial Hospital, Lodz, Poland (T.R.); Sun Yat-sen University Cancer Center, Guangzhou (H.H.), Department of Hematology, First Affiliated Hospital of Zhejiang University College of Medicine, Hangzhou (J.J.), Department of Lymphatic Oncology, Beijing Cancer Hospital, Beijing (J.Z.), Department of Hematology, West China Hospital of Sichuan University, Chengdu (T.L.), and Department of Medical Oncology, Fudan University Shanghai Cancer Center, Shanghai (X.H.) - all in China; Nizhny Novgorod Region Clinical Hospital, Nizhny Novgorod (O.S.), the Cancer Research Center, Russian Academy of Medical Sciences-N.N. Blokhin Academy of Medical Science, Moscow (E.O.), and Federal Center of Heart, Blood, and Endocrinology, St. Petersburg (J.A.) - all in Russia; Department of Hematology, Cherkassy Regional Oncology Center, Cherkassy, Ukraine (H.P.); Department of Hematology, University Hospital Leuven, Leuven (G.V.), and Janssen Research and Development, Beerse (H.V.) - both in Belgium; Department of Medicine, Faculty of Medicine Siriraj Hospital, Mahidol University, Bangkok, Thailand (N.S.); Hospital das Clinicas da Faculdade de Medicina da USP, São Paolo (J.P.); Department of Internal Medicine I, Division of Oncology, Medical University of Vienna, Vienna General Hospital, Vienna (J.D.); Department of Internal Medicine Hematology and Oncology, Faculty Hospital Brno, Brno, Czech Republic (J.M.); Department of Chemotherapy, Tokyo Metropolitan Cancer and Infectious Diseases Center, Komagome Hospital, Tokyo (R.O.); Janssen Research and Development, Raritan, NJ (L.P.); Janssen Research and Development, Janssen-Cilag, High Wycombe, United Kingdom (B.R.); and Oncology Institute of Southern Switzerland, Ospedale San Giovanni, Bellinzona (F.C.). 
1. Herrmann A, Hoster E, Zwingers T, et al. Improvement of overall survival in advanced stage mantle cell lymphoma. J Clin Oncol 2009;27:511-8.

2. Vose JM. Mantle cell lymphoma: 2013 update on diagnosis, risk-stratification, and clinical management. Am J Hematol 2013;88:1082-8.

3. Williams ME. Transplantation for mantle cell lymphoma: is it the right thing to do? Hematology Am Soc Hematol Educ Program 2013;2013:568-74.

4. Dreyling M, Geisler C, Hermine O, et al. Newly diagnosed and relapsed mantle cell lymphoma: ESMO clinical practice guidelines for diagnosis, treatment and followup. Ann Oncol 2014;25:Suppl 3:iii83-iii92. 5. McKay P, Leach M, Jackson R, Cook G, Rule S. Guidelines for the investigation and management of mantle cell lymphoma. Br J Haematol 2012;159:405-26.

6. NCCN clinical practice guidelines in oncology: non-Hodgkin's lymphomas, version 5. Fort Washington, PA: National Comprehensive Cancer Network, 2014 (http://www.nccn.org/professionals/ physician_gls/f_guidelines.asp).

7. Howard OM, Gribben JG, Neuberg DS, et al. Rituximab and CHOP induction therapy for newly diagnosed mantle-cell lymphoma: molecular complete responses are not predictive of progression-free survival. J Clin Oncol 2002;20:1288-94.

8. Velcade (bortezomib) for injection: for subcutaneous or intravenous use (package insert). Revision 17. Cambridge, MA Millennium Pharmaceuticals, October 2014 (http://www.velcade.com/files/pdfs/ velcade_prescribing_information.pdf).

9. Fisher RI, Bernstein SH, Kahl BS, et al. Multicenter phase II study of bortezomib in patients with relapsed or refractory mantle cell lymphoma. J Clin Oncol 2006; 24:4867-74.

10. Goy A, Bernstein SH, Kahl BS, et al. Bortezomib in patients with relapsed or refractory mantle cell lymphoma: updated time-to-event analyses of the multicenter phase 2 PINNACLE study. Ann Oncol 2009;20:520-5.

11. Kane RC, Dagher R, Farrell A, et al. Bortezomib for the treatment of mantle cell lymphoma. Clin Cancer Res 2007;13: 5291-4.

12. Mounier N, Ribrag V, Haioun C, et al. All B lymphoma subtypes do not share similar outcome after frontline R-CHOP plus bortezomib treatment: a randomized phase 2 trial from the Groupe d'etude des lymphomes de l'adulte (GELA). Ann Oncol 2008;19:Suppl 4. abstract.

13. Cheson BD, Pfistner B, Juweid ME, et al. Revised response criteria for malignant lymphoma. J Clin Oncol 2007;25:579-86. 14. Common Terminology Criteria for Adverse Events v3.0 (CTCAE). Bethesda, MD National Cancer Institute (http://ctep.cancer .gov/protocolDevelopment/electronic_ applications/docs/ctcaev3.pdf).
15. Determann O, Hoster E, Ott G, et al. $\mathrm{Ki}-67$ predicts outcome in advanced-stage mantle cell lymphoma patients treated with anti-CD20 immunochemotherapy: results from randomized trials of the European MCL Network and the German Low Grade Lymphoma Study Group. Blood 2008;111:2385-7.

16. Garcia M, Romaguera JE, Inamdar KV, Rassidakis GZ, Medeiros LJ. Proliferation predicts failure-free survival in mantle cell lymphoma patients treated with rituximab plus hyperfractionated cyclophosphamide, vincristine, doxorubicin, and dexamethasone alternating with rituximab plus high-dose methotrexate and cytarabine. Cancer 2009;115:1041-8.

17. Goy A, Bernstein SH, McDonald A, et al. Potential biomarkers of bortezomib activity in mantle cell lymphoma from the phase 2 PINNACLE trial. Leuk Lymphoma 2010;51:1269-77.

18. Tiemann M, Schrader C, Klapper W, et al. Histopathology, cell proliferation indices and clinical outcome in $304 \mathrm{pa}-$ tients with mantle cell lymphoma (MCL): a clinicopathological study from the European MCL Network. Br J Haematol 2005; 131:29-38.

19. Hoster E, Dreyling M, Klapper W, et al. A new prognostic index (MIPI) for patients with advanced-stage mantle cell lymphoma. Blood 2008;111:558-65. [Erratum, Blood 2008;111:5761.]

20. Hoster E, Klapper W, Hermine O, et al. Confirmation of the mantle-cell lymphoma International Prognostic Index in randomized trials of the European MantleCell Lymphoma Network. J Clin Oncol 2014;32:1338-46.

21. Kluin-Nelemans HC, Hoster E, Hermine $\mathrm{O}$, et al. Treatment of older patients with mantle-cell lymphoma. N Engl J Med 2012;367:520-31.

22. Flinn IW, van der Jagt R, Kahl BS, et al. Randomized trial of bendamustinerituximab or R-CHOP/R-CVP in first-line treatment of indolent NHL or MCL: the BRIGHT study. Blood 2014;123:2944-52. 23. Lenz G, Dreyling M, Hoster E, et al. Immunochemotherapy with rituximab and cyclophosphamide, doxorubicin, vincristine, and prednisone significantly improves response and time to treatment failure, but not long-term outcome in patients with previously untreated mantle cell lymphoma: results of a prospective randomized trial of the German Low Grade Lymphoma Study Group (GLSG). J Clin Oncol 2005;23:1984-92.

24. Rummel MJ, Niederle N, Maschmeyer $\mathrm{G}$, et al. Bendamustine plus rituximab versus CHOP plus rituximab as first-line treatment for patients with indolent and mantle-cell lymphomas: an open-label, multicentre, randomised, phase 3 noninferiority trial. Lancet 2013;381:1203-10. [Erratum, Lancet 2013;381:1184.]

25. Ribrag V, Gisselbrecht C, Haioun C, et al. Efficacy and toxicity of 2 schedules of frontline rituximab plus cyclophosphamide, doxorubicin, vincristine, and prednisone plus bortezomib in patients with B-cell lymphoma: a randomized phase 2 trial from the French Adult Lymphoma Study Group (GELA). Cancer 2009;115: 4540-6.

26. Furman RR, Martin P, Ruan J, et al. Phase 1 trial of bortezomib plus R-CHOP in previously untreated patients with aggressive non-Hodgkin lymphoma. Cancer 2010;116:5432-9.

27. Ruan J, Martin P, Furman RR, et al. Bortezomib plus CHOP-rituximab for previously untreated diffuse large B-cell lymphoma and mantle cell lymphoma. J Clin Oncol 2011;29:690-7.

28. O'Connor OA, Moskowitz C, Portlock $\mathrm{C}$, et al. Patients with chemotherapy-refractory mantle cell lymphoma experience high response rates and identical progression-free survivals compared with patients with relapsed disease following treatment with single agent bortezomib: results of a multicentre Phase 2 clinical trial. Br J Haematol 2009;145:34-9.

29. Broyl A, Corthals SL, Jongen JL, et al. Mechanisms of peripheral neuropathy associated with bortezomib and vincristine in patients with newly diagnosed multiple myeloma: a prospective analysis of data from the HOVON-65/GMMG-HD4 trial. Lancet Oncol 2010;11:1057-65

30. Cavaletti G, Jakubowiak AJ. Peripheral neuropathy during bortezomib treatment of multiple myeloma: a review of recent studies. Leuk Lymphoma 2010;51:1178-87. 31. Moreau P, Pylypenko H, Grosicki S, et al. Subcutaneous versus intravenous administration of bortezomib in patients with relapsed multiple myeloma: a randomised, phase 3, non-inferiority study. Lancet Oncol 2011;12:431-40.

32. Barbee MS, Harvey RD, Lonial S, et al. Subcutaneous versus intravenous bortezomib: efficiency practice variables and patient preferences. Ann Pharmacother 2013; 47:1136-42.

33. Lonial S, Waller EK, Richardson PG, et al. Risk factors and kinetics of thrombocytopenia associated with bortezomib for relapsed, refractory multiple myeloma. Blood 2005;106:3777-84.

34. Lonial S, Richardson PG, San Miguel J, et al. Characterisation of haematological profiles and low risk of thromboembolic events with bortezomib in patients with relapsed multiple myeloma. Br J Haematol 2008;143:222-9.

35. Murai K, Kowata S, Shimoyama T, et al. Bortezomib induces thrombocytopenia by the inhibition of proplatelet formation of megakaryocytes. Eur J Haematol 2014;93: 290-6.

36. Shi DS, Smith MC, Campbell RA, et al. Proteasome function is required for platelet production. J Clin Invest 2014;124:3757-66.

Copyright (@) 2015 Massachusetts Medical Society. 